\title{
Texture Feature Extraction for Identification of Medicinal Plants and Comparison of Different Classifiers
}

\author{
C. H. Arun \\ Department of Computer Science \\ NMC College \\ Marthandam, India
}

\author{
W. R. Sam Emmanuel \\ Department of Computer Science \\ NMC College \\ Marthandam, India
}

\author{
D. Christopher Durairaj \\ Department of Computer Science \\ VHNSN College \\ Virudhunagar, India
}

\begin{abstract}
This paper presents an automated system for recognizing the medicinal plant leaves that are taken from the suburbs of the western ghats region. The dataset comprises of 250 different leaf images, of five species. Texture analyses of the leaf images have been done in this work using the feature computation. The features include grey textures, grey tone spatial dependency matrices(GTSDM) and Local Binary Pattern(LBP) operators. For each leaf image, a feature vector is generated from the statistical values. $70 \%$ of the images in the dataset are the training dataset and the rest are included in the test set. Six different classifiers are used to classify the plant leaves based on feature values. When features are combined without any preprocessing, it yielded a classification performance of $94.7 \%$.
\end{abstract}

\section{General Terms:}

image processing, pattern recognition

\section{Keywords:}

image classification, texture features, plant identification

\section{INTRODUCTION}

Herbal medicines are gaining popularity worldwide as they are safe to human health and affordable. World Health Organization estimates that $80 \%$ of people in Asia and Africa rely on herbal medicines for some aspects of their primary health care [1]. There are diverse medicinal plant varieties in lush forests of southern india which are used as medicines to a number of sicknesses like common cold, allergies etc. Precise identification of the respective plant leaf is vital in treating the patients. The present work explores the use of texture features to identify the appropriate medicinal leaves automatically.

Textures are a pattern of non-uniform spatial distribution of differing image intensities [11], which focus mainly on the individual pixels that make up an image. Texture is defined by quantifying the spatial relationship between materials in an image. Texture analyses is done by using various approaches like statistical, fractal, and structural. Statistical type includes techniques like grey-level histogram, grey-level co-occurence matrix [20], local binary pattern operator, auto-correlation features and power spectrum.

Grey texture features or the first order statistical features are used in image classification [15]. Haralick et al [14], described texture features based on grey-level co-occurence
matrix(GLCM) and are used in various applications including Land cover classification [22]. Ojala et al [19] used texture feature based on local binary pattern(LBP).

Textures are useful in identifying plant leaves without the need for an expert. [26][21][12]. Plant leaves are recognized based on the features of leaf images [7]. Classification of leaf epidermis microphotographs is done using texture features [23]. Basavaraj et al [3], Sandeep et al [24] identified medicinal plant leaves using textures.

Classification approaches like Stochastic Gradient Descent(SGD) [25][18], kNearest Neighbour(kNN) [4][2], Support Vector Machines(SVM) [10], Decision Trees(DT) [5], Extra Trees(ET) [13], Random Forests(RF) [8][6] can be used as Image classifiers.

The objective of the present work is to compute texture features based on grey-level, the Grey Tone Spatial Dependency Matrix(GTSDM) and the Local Binary Pattern(LBP) operators and to obtain the best combination of these, for automatically identifying the right medicinal plant.

Five medicinal plant leaves are considered and few of their medicinal uses are given below. A sample of the medicinal plant leaf dataset from different plant leaves are given in Fig. 1.

(0) Desmodium gyrans - Antidote for snake poison, effective for heart diseases, rhematic complaints, Diabetes and skin ailments.

(1) Butea monosperma - promotes diuresis and anthelmintic, treats leucorrhea and diabetes.

(2) Malpighia glabra - help lower blood sugar, increases collagen and elastin production, treats diarrhea, dysentery, and liver problems.

(3) Helicteres isora - help treat intestinal complaints, colic pains and flatulence.

(4) Gymnema sylvestre - suppresses the sensation of sweet, anti-diabetic.

The rest of the paper is organized as follows: Methodology of the work is discussed in section 2. This section includes the details of texture based image classification and the description of the dataset. Design and implementation of the work are discussed in section 3. The results and discussion are given in section 4 , which is followed by the conclusion. 

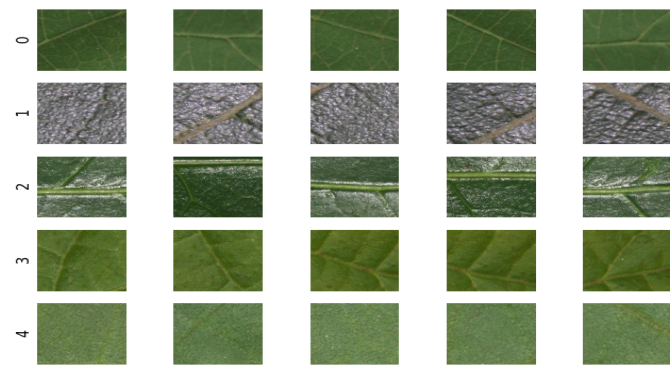

Fig. 1. Leaf images of Desmodium gyrans (row 0), Butea monosperma (row 1), Malpighia glabra (row 2), Helicteres isora (row 3)and Gymnema sylvestre (row 4)

\section{METHODOLOGY}

\subsection{Texture Features}

The different Texture features namely, Grey-level, GTSDM based second-order and LBP features are briefly presented in this section.

2.1.1 Grey Textures. One of the simplest texture descriptors is first-order statistical central moments which are also referred as Gray level features. They include mean, variance, skewness and standard deviation. First order features do not take relative layout between gray values into account.

The $x_{i}$ be a random variable which denotes the gray levels in an image and $\mathrm{L}$ be the number of distinct gray levels, then $P\left(x_{i}\right)$ is defined as the probability of occurence of pixels with intensity $x_{i}$ as in Eqn.(1).

$$
P\left(x_{i}\right)=\frac{k}{N}
$$

where $k$ is the number of pixels with grey level $x_{i}$ and $\mathrm{N}$ is the total number of pixels in the region.

Mean $\left(\mu_{0}\right)$, the first of the grey-level feature, which averages the gray levels of the image is defined as in Eqn.(2).

$$
\mu_{0}=\sum_{i=0}^{L-1} x_{i} P\left(x_{i}\right)
$$

Variance $\left(\mu_{2}\right)$ is referred as the second moment which is a measure of gray-level contrast that are used to describe the descriptors of relative smoothness. Variance is defined as in Eqn.(3). Variance values tend to be larger for images with values.

$$
\mu_{2}=\sum_{i=0}^{L-1}\left(x_{i}-\mu_{0}\right) P\left(x_{i}\right)
$$

The third moment is a measure of skewness $\left(\mu_{3}\right)$ of the histogram as in Eqn.(4). The third moment will be of use only when the variations between measurements are too large.

$$
\mu_{3}=\sum_{i=0}^{L-1}\left(x_{i}-\mu_{0}\right)^{2} P\left(x_{i}\right)
$$

Standard Deviation $(\sigma)$ can also be used as a measure of texture and is defined as in Eqn.(5).

$$
\sigma=\sqrt{\frac{1}{L} \sum_{i=0}^{L-1}\left(P\left(x_{i}\right)-\mu_{0}\right)^{2}}
$$

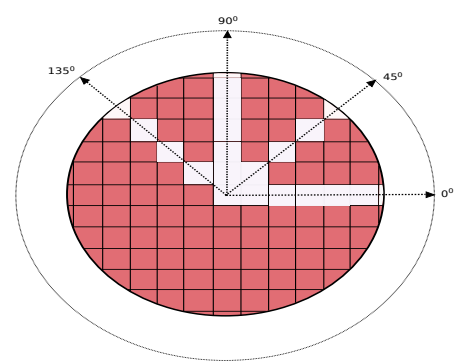

Fig. 2. The $\theta$ orientations for feature computation

2.1.2 GTSDM Based Textures. Grey Tone Spatial Dependency Matrix(GTSDM) features are second-order features and are usually more sensitive and intuitive than first-order measures. GTSDM texture features are based on the GTSD matrix. The texture is related with the statistical distribution of gray tones. The GTSDM is a tabulation of probability of grey levels $i$ occuring in an image at a distance $d$ in angle $\theta$ from grey level $j$ at points from $p_{1}\left(x_{1}, y_{1}\right)$ to $p_{2}\left(x_{2}, y_{2}\right)$. These probabilities create the co-occurence matrix as of Eqn.(6).

$$
G=P(i, j \mid d, \theta)
$$

The distance and the angle are obtained as given in Eqn. (7) and Eqn. (8) and the different directions of adjacency for calculation the texture feature is given in Fig. 2

$$
\begin{gathered}
d\left(p_{1}, p_{2}\right)=\sqrt{\left(x_{2}-x_{1}\right)^{2}+\left(y_{2}-y_{1}\right)^{2}} \\
\theta\left(p_{1}, p_{2}\right)=\tan ^{-1} \frac{\left(y_{2}-y_{1}\right)}{\left(x_{2}-x_{1}\right)}
\end{gathered}
$$

By computing the frequency of one grey tone to the neighbourhood of others, a $k \times k$ matrix is formed. The frequencies of the reference and neighbour grey-tones constituting a pair in the colour image is computed. The $(i, j)^{t h}$ element of GTSDM matrix $\mathrm{G}$ is computed by finding the frequency of the neighbour grey-tone $j$ occuring near the reference grey-tone $i$ within the colour image. In order to make the generated matrix independent of the direction, the matrix $\mathrm{G}$ is transposed $\left(G^{\prime}\right)$ to make it symmetrical(S), which is defined in Eqn.(9).

$$
S=G+G^{\prime}
$$

The Symmetrical GTSDM is then normalised by dividing each element by the sum of all the elements as in Eqn.10 to form $S_{\theta}$, where $\theta$ is the angle which varies as $0,45,90$ and 135 as shown in Fig. 2. This represents the directions along horizontal $\left(\theta=0^{\circ}\right)$, right diagonal $\left(\theta=45^{\circ}\right)$, vertical $\left(\theta=90^{\circ}\right)$ and the left-diagonal $\left(\theta=135^{\circ}\right)$.

$$
S_{\theta}=\frac{S}{\sum_{i} \sum_{j} S_{i j}}
$$

The statistically derived texture features [14] using co-occurrence matrix identify some structural aspects of the arrangement of probabilities within a matrix, which in turn reflect some characteristics of the texture. Entropy texture features are considered from the GTSTD matrix. Entropy $(\epsilon)$ is a measure of uncertainity associated with the random occurences, normally considered as the measure of 'disorder'. Homogeneous scenes has a high entropy and inhomogeneous scenes have low 


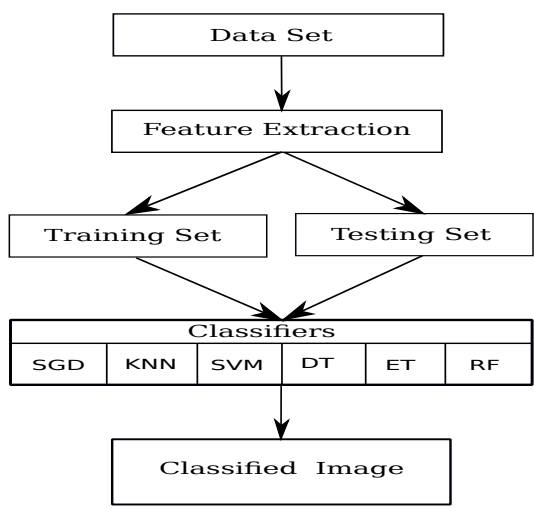

Fig. 3. Block diagram for Classification

entropy. Entropy is defined as in Eqn.(11). The term $S_{i j}$ in the equation represent the element $(i, j)$ of a normalised symmetrical GTSDM and $k$ the number of grey levels.

$$
\epsilon=-\sum_{i=1}^{k} \sum_{j=1}^{k} S_{i j} \log \left[S_{i j}\right]
$$

2.1.3 LBP Based Textures. The Local Binary Pattern(LBP)[19] features are texture operators which are used in diverse applications. They are known for its invariance to local grayscale variations and monotonic photometric changes and its high descriptive power.

The image is assumed to be constituted by micropatterns of the input image such as spots, lines, flat areas, and edges. The value is calculated on the basis of the circular neighborhood of $\mathrm{P}$ pixels of gray levels $q_{p}$ and radius $r$ around the central pixel of gray value $q_{c}$. The LBP is defined as Eqn. (12).

$$
\begin{aligned}
& L B P(P, r)=\sum_{p=1}^{P-1} s\left(q_{p}-q_{c}\right) 2^{p} \\
& \text { where } s(x)= \begin{cases}1 & \text { if } x \geq 0 \\
0 & \text { otherwise }\end{cases}
\end{aligned}
$$

The extracted binary codes are used to describe the texture patterns in the image. The mean of the LBP histogram $\left(\mu_{L}\right)$ is considered as one of the feature.

\subsection{Texture Based Image Classification}

Texture based image classification involves deciding the most pertinent texture category of the observed image[14]. Classification here would mean the machine language classification of the different classes and not the linnaean taxonomic system of plant classification[9]. When the priori knowledge of the established classes are available and the texture features are extracted, the given image could be classified to the appropriate class.

The texture class $i$ consisting of a set of $n$ images can be represented as

$$
T_{i}=\left\{t_{i 1}, t_{i 2}, \cdots, t_{i n}\right\}
$$

where $t_{i j}$ is the member image.

Various classifiers used in this work are SGD, kNN, SVM, DT, ET and RF. SGD is an approach to discriminative learning of linear classifiers under convex loss functions. kNN computes the distance between a test point and all points in the training set. SVM are supervisor learning methods which are similar to SGDs and these are effective in high dimensional feature spaces.
DTs are a non-parametric supervised learning methods which creates a model that predicts the value of a target variable by learning simple decision rules learned from the data features. RF are average ensemble methods which combines and average the predictions of several models. ET implements a meta estimator that fits a number of extra-tree on various sub-samples of the dataset and use averaging to improve the predictive accuracy and control over-fitting.

The classifier places all of the $\mathrm{N}$ training images in a vector space with $\mathrm{N}$ dimensions, based on the features extracted from the image. When a new uncategorized test image is placed in that vector space, the best suited image is found using the pairwise-distance function. The pairwise-distance between two images are calculated as given in the Eqn. (13) where $d 1$ an $d 2$ are the two images. When the distance between $d 1$ and $d 2$ are the smallest, more similar images are obtained.

$$
\operatorname{distance}(d 1, d 2)=\frac{1}{L}
$$

where $L$ is the number of matching images.

The test image is estimated to belong to a specific category, after generation of its features. Fig 3 shows the block diagram for classification.

\subsection{Dataset}

The digital images of medicinal plant leaves are collected from the suburbs of western ghats region. The collection process is done by taking digital photograph of the abaxial portions of the medicinal plant leaf in the digital form using the digital camera Canon EOS 1000D. The digital colour images are transferred to the computer in the uncompressed JPEG format with $3420 \mathrm{x}$ $4320 \times 3$ size. The working dataset is formed by dividing the images into a size of $50 \times 50 \times 3$ and forming a set total of 250 images, 50 images each from 5 leaves and stored as JPEG format. The training and the testing sets are formed from the image datasets.

\section{IMPLEMENTATION}

The implementation of the proposed method is done using Python in the Enthought Python Distribution(EPD 7.3-1) environment with the libraries scipy, numpy, scikit-learn, in the ubuntu 10.04 linux platform.

The various stages of identifying the medicinal leaves are explained in detail below. The functional block diagram is given here in Fig. 4.

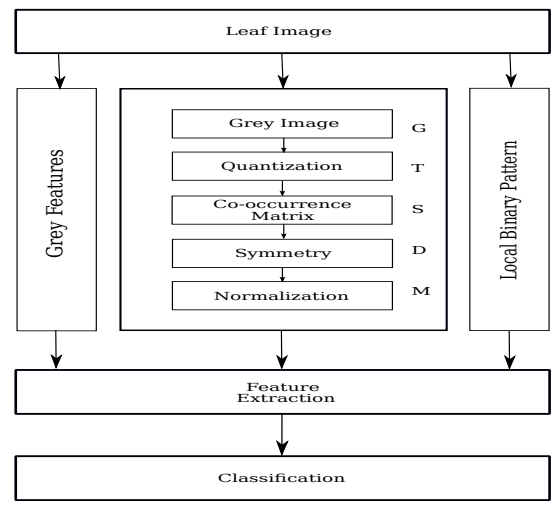

Fig. 4. Functional Diagram of the Leaf classification system 


\subsection{Leaf' statistical features}

The medicinal leaf image is available as an $m \times n \times c$ colour image LI.

$$
L I=\left[\begin{array}{ccccc}
c_{11} & c_{12} & c_{13} & \cdots & c_{1 n} \\
c_{21} & c_{22} & c_{23} & \cdots & c_{2 n} \\
\vdots & \vdots & \vdots & \ddots & \vdots \\
c_{m 1} & c_{m 2} & c_{m 3} & \cdots & c_{m n}
\end{array}\right]
$$

where $c_{i j}$ represents the colour channels of the image.

The grey-features using mean, variance, skewness and standard deviation are calculated directly from the original image LI as in Eqns. (2),(3),(4) and (5).

The GTSDM deals only with grey-tone scales and so the given input image LI is converted into a $m \times n$ grey-scale image $(G I)$

$$
G I=\left[\begin{array}{ccccc}
g_{11} & g_{12} & g_{13} & \cdots & g_{1 n} \\
g_{21} & g_{22} & g_{23} & \cdots & g_{2 n} \\
\vdots & \vdots & \vdots & \ddots & \vdots \\
g_{m 1} & g_{m 2} & g_{m 3} & \cdots & g_{m n}
\end{array}\right]
$$

where $g_{i j}$ represents the grey-tones of the image.

As quantization gives the range of reflectance values in a particular spectral band and the data reduces the radioactive sensitivity of the data, a two-bit image quantization $(Q I) m \times n$ matrix is obtained

$$
Q I=\left[\begin{array}{ccccc}
q_{11} & q_{12} & q_{13} & \cdots & q_{1 n} \\
q_{21} & q_{22} & q_{23} & \cdots & q_{2 n} \\
\vdots & \vdots & \vdots & \ddots & \vdots \\
q_{m 1} & q_{m 2} & q_{m 3} & \cdots & q_{m n}
\end{array}\right]
$$

where $q_{i j}$ represents the quantized values of the image. The feature computations requires the generation of the co-occurrence matrix from the quantization matrix, which is the GTSDM.

The $\operatorname{GTSDM}\left(M_{\theta}\right)$ is calculated along the different directions as given in Fig. 2 with $\theta$ as $0^{\circ}, 45^{\circ}, 90^{\circ}$ and $135^{\circ}$ with the order $k \times k$. The $M_{\theta}$ is in the form

$$
M_{\theta}=\left[\begin{array}{ccccc}
f_{11}^{\theta} & f_{12}^{\theta} & f_{13}^{\theta} & \cdots & f_{1 k}^{\theta} \\
f_{21}^{\theta} & f_{22}^{\theta} & f_{23}^{\theta} & \cdots & f_{2 k}^{\theta} \\
\vdots & \vdots & \vdots & \ddots & \vdots \\
f_{k 1}^{\theta} & f_{k 2}^{\theta} & f_{k 3}^{\theta} & \cdots & f_{k k}^{\theta}
\end{array}\right]
$$

where $f_{i j}^{\theta}$ represents the co-occurences on $\theta$ directions of the image.

Here the value of $\mathrm{k}$ is determined from the maximum value of the elements of $M_{\theta}$

$$
\text { i.e. } k=\max \left(q_{i j}\right)
$$

where $i=1,2, \cdots m$, and $j=1,2, \cdots n$

The $M_{\theta}$ generated is a square matrix which has dimensions equalling the number of gray levels in the image and the elements correspond to the relative frequencies of the occurrence of pairs of gray level pixels in the image. The $M_{\theta}$ is transposed to make the symmetrical matrix $\left(S_{\theta}\right)$. The normalized form of $S_{\theta}$ is $N_{\theta}^{T}$, which can be defined as

$S_{\theta}$ is normalized to get $N_{\theta}$

$$
N_{\theta}=\left[\begin{array}{ccccc}
n_{11}^{\theta} & n_{12}^{\theta} & n_{13}^{\theta} & \cdots & n_{1 k}^{\theta} \\
n_{21}^{\theta} & n_{22}^{\theta} & n_{23}^{\theta} & \cdots & n_{2 k}^{\theta} \\
\vdots & \vdots & \vdots & \ddots & \vdots \\
n_{k 1}^{\theta} & n_{k 2}^{\theta} & n_{k 3}^{\theta} & \cdots & n_{k k}^{\theta}
\end{array}\right]
$$

where $n_{i j}^{\theta}$ represents the normalized co-occurences on $\theta$ directions.
The second-order statistical features $\left(F_{i}^{\theta}\right)$ are computed from the normalised image $\left(N_{\theta}\right)$ for each $\theta$. The mean feature(MF) vector, as in Eqn.14, is obtained after averaging the feature along all the directions.

$$
M F=\frac{1}{4} \sum_{\theta} \sum_{i} F_{i}^{\theta}
$$

The mean feature values are used for classification in regard to GTSDM features.

The local binary pattern operator also deals with the grey-tone image and the image LI is used to calculate the feature. The local binary pattern operator used 8 neighbours and a unit radius for computation of the pattern. From the computed LBP histogram image as from Eqn. (12) the mean of the histogram is calculated $\left(\mu_{L}\right)$ and used as a feature.

\subsection{Feature extraction and classification}

Various features from first order statistics, GTSDM feature and LBP are computed. They are mean $\left(\mu_{0}\right)$, variance $\left(\mu_{2}\right)$, skewness $\left(\mu_{3}\right)$, standard deviation $(\sigma)$, GTSDM Entropy $(\epsilon)$ and LBP mean $\left(\mu_{L}\right)$. Different combinations of the computed features are worked out to find out the best feature combination that will provide the better classification. Initially feature combinations from category $\mathrm{C} 1, \mathrm{C} 2, \ldots \mathrm{C} 5$ are worked out with few pre-processing filters and then without preprocessing.

The texture features are combined into categories as given below. The category combinations chosen are $\mathrm{C} 1\left(\sigma \bullet \mu_{3}\right), \mathrm{C} 2\left(\mu_{2} \bullet \mu_{3}\right)$, $\mathrm{C} 3\left(\mu_{0} \bullet \mu_{3}\right), \mathrm{C} 4\left(\mu_{0} \bullet \mu_{3} \bullet \epsilon\right)$ and C5 $\left(\mu_{0} \bullet \mu_{3} \bullet \epsilon \bullet \mu_{2} \bullet \sigma \bullet \mu_{L}\right)$. The preprocessing filters that are used with the categories are:

Histogram Equalisation(HE)

Adaptive Histogram Equalisation(AHE)

Contrast Limited Adaptive Histogram

Equalisation(CLAHE)

Local Binary Pattern(LBP)

The classification stage has two phases:training and testing. During the training phase, $70 \%$ of the dataset samples are used as the training set. The texture features are calculated from the given set of training images and these features are used to train the classifier. During the testing phase, the remaining 30\% of the dataset are used as the testing set. The texture features are calculated for the test images and these are the input image to the classifier which finds out the probable plant leaf class category.

\section{RESULTS AND DISCUSSION}

The experimentation has been conducted on medicinal plant leaf database of 5 leaves with 50 sample images in each. Features are computed for each of the 250 medicinal leaf images. The sample feature values computed on images of each leaves are listed in Table 1 .

The performance measure for each classifiers are calculated which are presented in tabular and graphical representations. This experiment identified the respective medicinal plant leaves using performance accuracy as the tool.

A sample preprocessed image set consisting of the original image, Histogram equalization image, Adaptive Histogram image, Contrast Limited Adaptive Histogram Equalization image, Local Binary Pattern (LBP) operator image are shown in Fig. 5.

\subsection{Preprocessing with category $\mathrm{C1}$}

When the preprocessing test is done with the category $\mathrm{C} 1$, the best performance obtained for $\mathrm{HE}$ and AHE is $41.3 \%$ with RF, for CLAHE is $38.7 \%$ with RF and for LBP is $49.3 \%$ with $\mathrm{kNN}$. In this case, LBP presents $49.3 \%$ with $\mathrm{kNN}$ classification, which is the best performance from among the filters. The detailed 

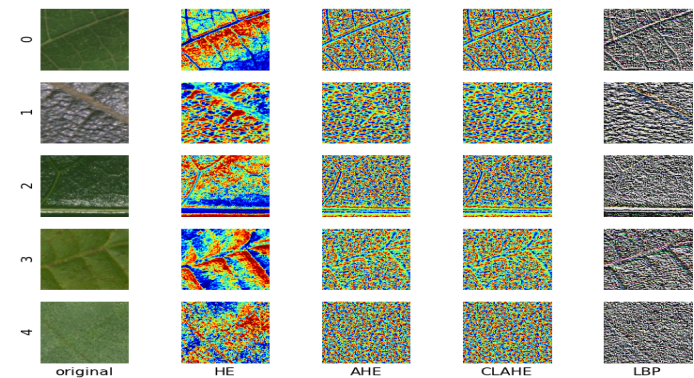

Fig. 5. Processed image sample set

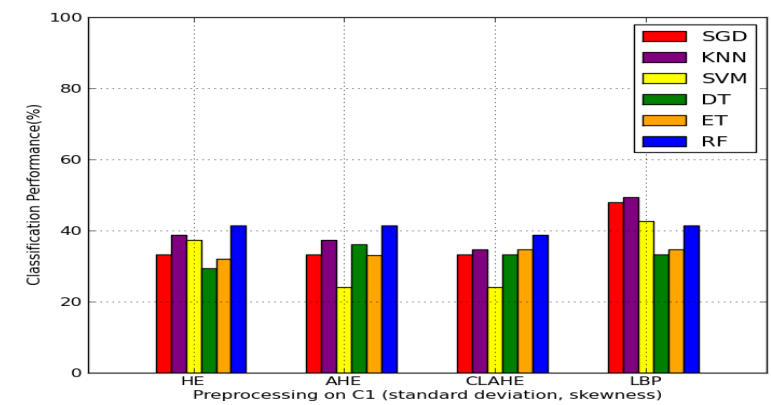

Fig. 6. Classification accuracy with Category $\mathrm{C1}$ on different filters

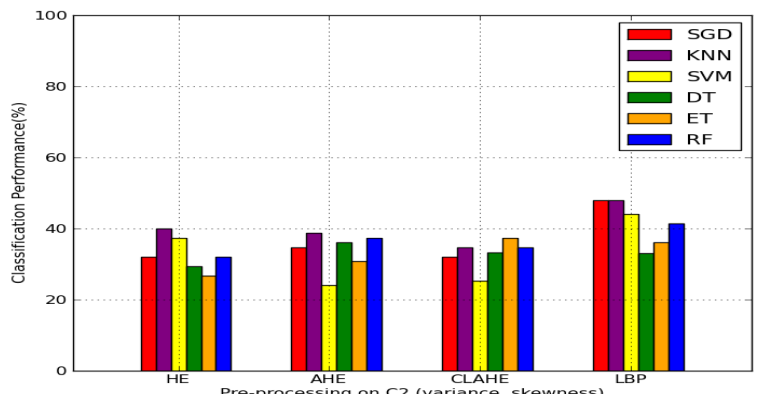

Fig. 7. Classification accuracy with Category C2 on different filters

accuracy performance values are displayed in the Table 2 and the values are plotted in Fig. 6 .

\subsection{Preprocessing with category $\mathrm{C} 2$}

When the preprocessing is done with the category $\mathrm{C} 2$, the highest performance obtained for $\mathrm{HE}$ is $40 \%$ with $\mathrm{kNN}$, for AHE is $38.7 \%$ with $\mathrm{kNN}$ and for CLAHE is $37.3 \%$ with ET and for LBP is $48.0 \%$ with SGD and kNN. In this case, LBP with SGD and kNN classification presents $48.0 \%$, which is the best performance. The detailed accuracy performance values are displayed in the Table 3 and the values are plotted in Fig. 7.

\subsection{Preprocessing with category $\mathrm{C3}$}

When the preprocessing test is carried with the category $\mathrm{C} 3$, the best performance obtained for HE is $33.3 \%$ with SGD, for AHE is $45.3 \%$ with SGD, for CLAHE is $45.3 \%$ with $\mathrm{kNN}$ and for LBP is $50.7 \%$ with ET. In category C3, LBP presents $50.7 \%$ with ET classification, which gives the best performance result among the

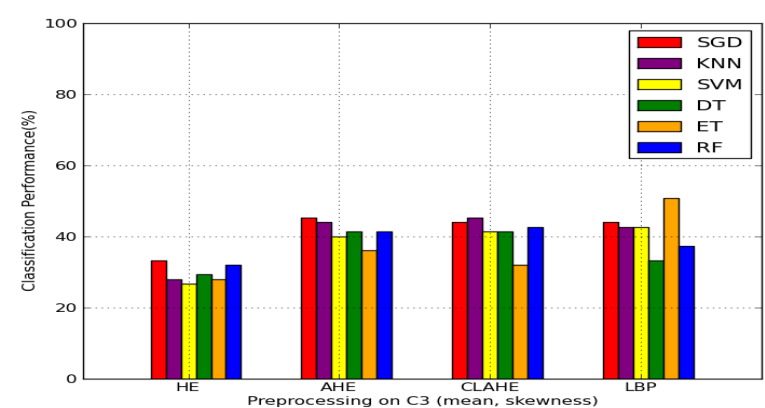

Fig. 8. Classification accuracy with Category C3 on different filters

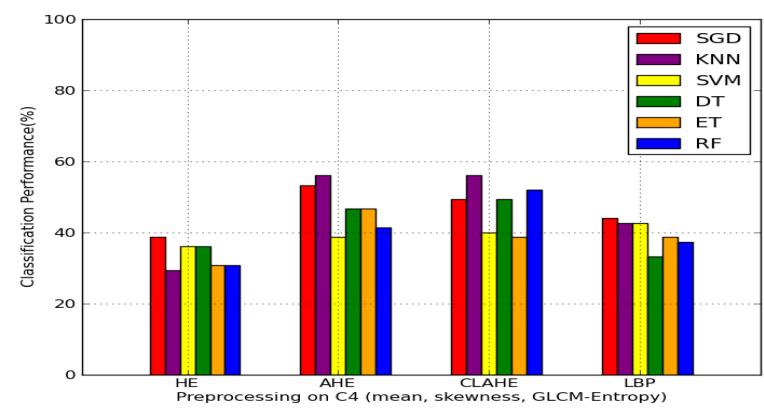

Fig. 9. Classification accuracy with Category $\mathrm{C} 4$ on different filters

different filters. The detailed accuracy performance values are displayed in the Table 4 and the values are plotted in Fig. 8.

\subsection{Preprocessing with category $\mathrm{C} 4$}

When the preprocessing test is done with the category $\mathrm{C} 4$, the best performance obtained for $\mathrm{HE}$ is $38.7 \%$ with SGD, for AHE and CLAHE is $56 \%$ with $\mathrm{kNN}$ and for LBP is $44.0 \%$ with SGD. In this case, AHE and CLAHE presents 56\% with kNN classification, which is the best performance here. The detailed accuracy performance values are displayed in the Table 5 and the values are plotted in Fig. 9.

\subsection{Preprocessing with category C5}

When the preprocessing test is performed with the category C5, the best result obtained for HE is $57.3 \%$ with RF, for AHE is $64 \%$ with $\mathrm{kNN}$ and CLAHE is $66.7 \%$ with $\mathrm{kNN}$ and for LBP is $57.3 \%$ with $\mathrm{kNN}$. In this case, CLAHE presents $66.7 \%$ with $\mathrm{kNN}$ classification, which is the best performance. The detailed accuracy performance values are displayed in the Table 6 and the values are plotted in Fig. 10.

\subsection{No Preprocessing(NP)}

It is observed that without preprocessing(NP) the best performance obtained is $88.0 \%$ with $\mathrm{kNN}$ for categories $\mathrm{C} 1$ and C2, $94.7 \%$ with DT for C3, $94.7 \%$ with kNN for C4, $94.7 \%$ with SGD for category C5. In this case, the best performance of $94.7 \%$ is achieved using the categories $\mathrm{C} 3, \mathrm{C} 4$ and $\mathrm{C} 5$. The detailed accuracy performance values are displayed in the Table 7 and the values are plotted in Fig. 11. 


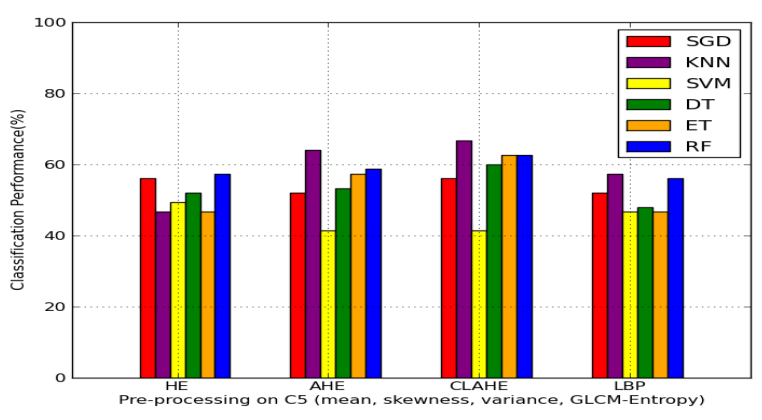

Fig. 10. Classification accuracy with Category $\mathrm{C} 5$ on different filters

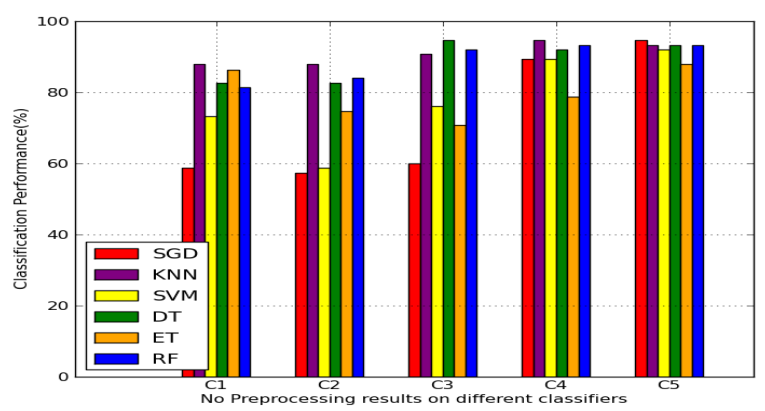

Fig. 11. 'No Preprocessing(NP)' classification accuracy on different categories

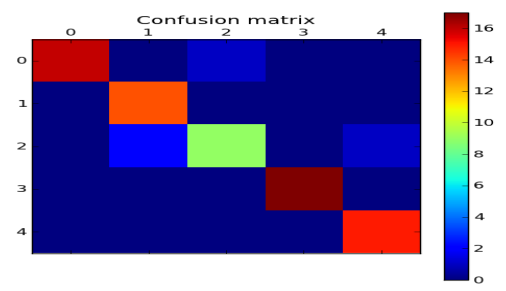

Fig. 12. Confusion matrix of $\mathrm{C} 3$ using Decision Tree classifier

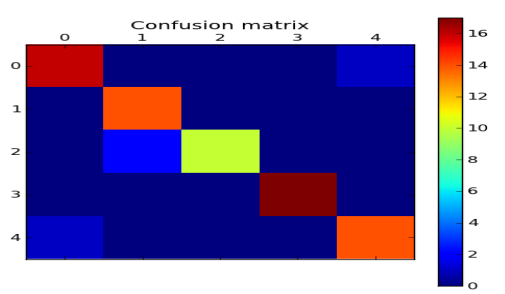

Fig. 13. Confusion matrix of $\mathrm{C} 4$ using $\mathrm{kNN}$ classifier

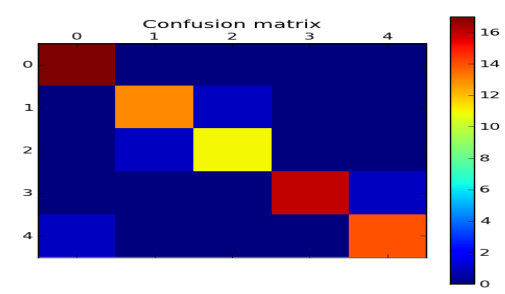

Fig. 14. Confusion matrix of $\mathrm{C} 5$ using SGD classifier

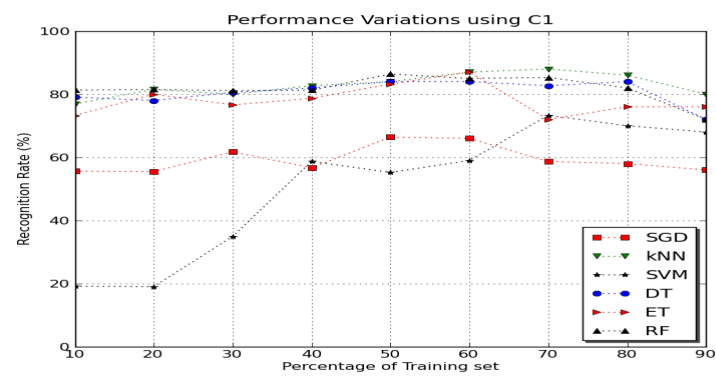

Fig. 15. Training and Testing possible variation performance for C1

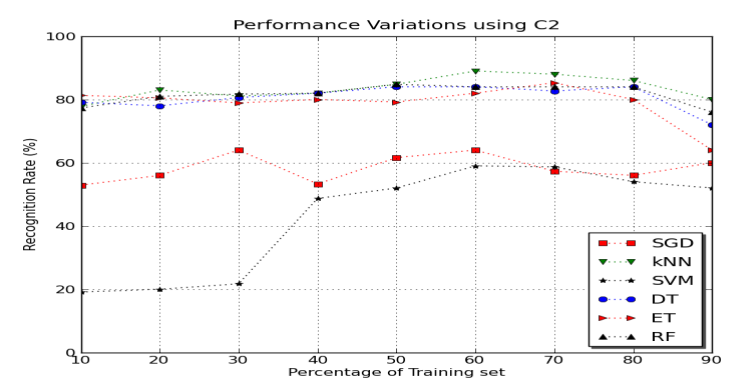

Fig. 16. Training and Testing possible variation performance for C2

\subsection{Training Set Vs Recognition Rate}

The performance evaluation is done for the 'No Preprocessing(NP)' category, because it's percentage of classification is more, than in all other categories. The original data set is fixed with 250 medicinal plant leaf images. The performance variations obtained for the different categories $\mathrm{C} 1$, $\mathrm{C} 2, \ldots, \mathrm{C} 5$ under the different Training/Testing Vs Recognition possibilities using the all the six classifiers SGD, kNN, SVM, DT, ET and RF, obtained from the selected individual plant leaf images, are given in Figures 15, 16, 17, 18 and 19 respectively. The above figures shows the relation between the percentage of samples for training in the medical leaf database and the recognition rate. It is also observed that in almost all the cases, the recognition system's performance gradually increases when the training data increases and dips down finally. It shows that the recognition rate is increasing when the training set is increasing upto a certain level. It is found from the above figure that with the training of $70 \%$ and with testing $30 \%$, the system performed well with the best recognition rate. The training and testing set are therefore selected with the splitup of $70 \%$ and $30 \%$ respectively. 


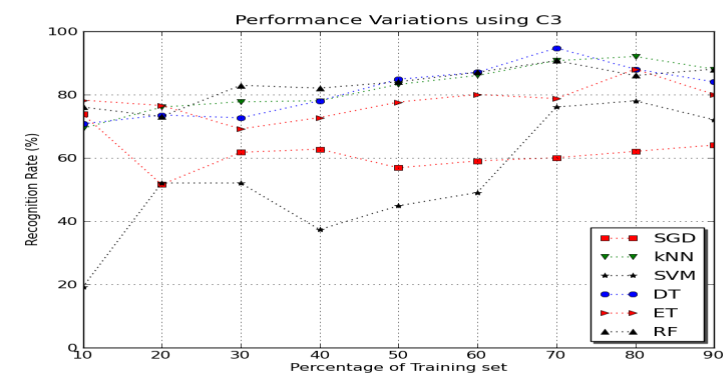

Fig. 17. Training and Testing possible variation performance for C3

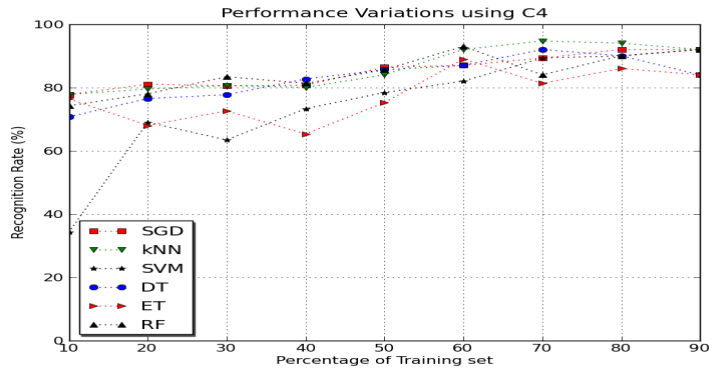

Fig. 18. Training and Testing possible variation performance for C4

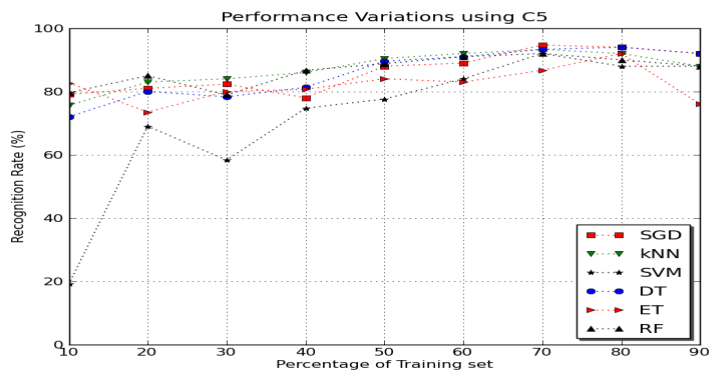

Fig. 19. Training and Testing possible variation performance for C5

\subsection{Performance Analysis}

The distribution of the testing set contains 75 of medicinal leaf images(ie. 30\% of the dataset) given as support in Fig. 20. The different recognition rate and error rate are calculated as in Eqn.(15) and Eqn.(16) respectively. The recognition rate is the measure of the percentage of the ratio between prediction and the support. They are calculated when the support is having 17 leaf images of leaf 0,14 leaf images of leaf 1,12 leaf images of leaf 2, 17 leaf images of leaf 3 and 15 leaf images of leaf 4 . The percentages of the leaf images taken for testing is displayed in Fig. 20. The correctly classified leaf images are presented in the prediction.

$$
\text { RecognitionRate }=\frac{\text { Prediction }}{\text { Support }}
$$

$$
\text { Error }=1-\text { RecognitionRate }
$$

The testing is done 25 times. In this process, the maximum classification performance of $94.7 \%$ is obtained though there

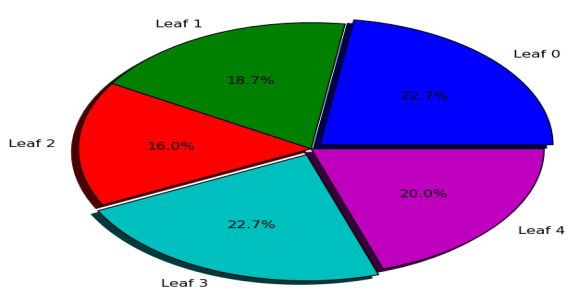

Fig. 20. Support for the test images

are some minor deviations in the other performance values. The confusion matrix also have some minor changes in every iterations. Most of the times the result is in the form of the confusion matrix shown in Fig. 12, Fig. 13, and Fig. 14 for categories $\mathrm{C} 3, \mathrm{C} 4$ and $\mathrm{C} 5$ respectively.

The decision tree(DT) classifier gives best performance in the No preprocessing C3 category with $100 \%$ classification for the medicinal plant leaves, leaf 1 , leaf 3 and leaf 4 , but the worst performance is produced for leaf 2 with $75 \%$ classification. The detailed recognition rate is shown in Table 8 .

The $\mathrm{kNN}$ classifier gives best performance in the No processing(NP) C4 category with $100 \%$ classification for the medicinal plant leaves, leaf 1 and leaf 3 , but the worst performance is performed for the leaf 2 with $83 \%$ classification. The detailed recognition rate is shown in Table 9.

The SGD classifier gives best performance in the No processing(NP) C5 category with $100 \%$ classification for the medicinal plant leaf, leaf 0 only, but the worst performance is performed for the leaf 2 with $92 \%$ classification. The detailed recognition rate is shown in Table 10.

The error rate is very high for the leaf 2 with $25 \%$ in Table 8 , $17 \%$ in Table 9 , and $8 \%$ in Table 10 . The best classification gives very small error rate for the medicinal plant leaf identification. It is necessary to reduce the error rate for better result. The reduction of error rate may depend on the selected features and the dataset.

Much research is done on plant leaf identification. It is imperative to have a further detailed study on the medicinal leaf identification because of it's importance pertaining to life. Table 11 shows the list of recognition rate of the proposed method and other methods introduced by other researchers. It is shown that the proposed approach gives better recognition rate than the others.

\section{CONCLUSION}

Herbal medicinal plant leaf identification using texture analyses is a sound method for plant identification. The texture analyses is done using grey textures, GTSDM and lbp texture combinations. The different category combinations using few preprocessing filter and without preprocessing are computed. The method of classifying without preprocessing performed better. The classification performance of $94.7 \%$ is obtained using Stochastic Gradient Descent, Decision Tree and kNN classifiers. Therefore it is concluded that the preprocessing approach is not suitable for the medicinal leaf identification. Instead of using preprocessing, the direct application of feature extraction with different categories produced better performance.

It is also observed that, choosing additional suitable feature might help in easier classification and identifation of leaf 2 from other selected leaves and may increase the recognition rate. The recognition rate and the error rate are compared with the existing methods. 


\section{ACKNOWLEGEMENTS}

Thanks are due to Dr. J.T.T. KUMARAN, Head, Department of Physics, Nesamony Memorial Christian College, Marthandam, for being a great source of encouragement.

\section{REFERENCES}

[1] World Health Organization(WHO) Traditional Medicine Fact Sheet $N^{o}$ 134. Technical report, WHO, Dec 2008.

[2] Giuseppe Amato and Fabrizio Falchi. kNN based image classification relying on local feature similarity. In Proceedings of the Third International Conference on Similarity Search and Applications SISAP '10, pages 101-108, 2010.

[3] Basavaraj S. Anami, Suvarna S. Nandyal, and A. Govardhan. A combined color, texture and edge features based approach for identification and classification of indian medicinal plants. International Journal of Computer Applications(0975-8887), 6(12):45-51, September 2010. Published By Foundation of Computer Science.

[4] A. Bosch, A. Zisserman, and X. Muoz. Scene classification using a hybrid generative/discriminative approach. IEEE Transactions on Pattern Analysis and Machine Intelligence, 30(4):712 - 727, April 2008.

[5] L. Breiman, J. H. Friedman, R. A. Olshen, and C. J. Stone. Classification and Regression Trees. Chapman Hall, New York, 1984.

[6] Leo Breiman. Random forests. Mach. Learn., 45(1):5-32, October 2001

[7] Jyotismita Chaki and Ranjan Parekh. Plant leaf recognition using shape based features and neural network classifiers. (IJACSA) International Journal of Advanced Computer Science and Application, 2(10):41-47, 2011.

[8] Jonathan Cheung-Wai Chan and Desir Paelinckx. Evaluation of randomforest and adaboost tree-based ensemble classification and spectral band selection for ecotope mapping using airborne hyperspectral imagery. Remote Sensing of Environment, 112(6):29993011, 16 June 2008. Elsevier.

[9] John Lee Comstock. An introduction to the study of botany: including a treatise on vegetable physiology, and descriptions of the most common plants in the middle and northern states. Robinson, Pratt Co., 1837.

[10] C. Cortes and V. Vapnik. Support-vector networks. Machine Learning, 20:273-297, 1995.

[11] Larry S. Davis and Larry S Davis. Image texture analysis techniques - a survey. In Digital Image Processing, Simon and, pages 189-201, 1980.

[12] Joo Batista Florindo and Odemir Martinez Bruno. Texture classification based on lacunarity descriptor. Image and Signal Processing: Lecture Notes in Computer Science, 7340:513-520, 2012.

[13] Pierre Geurts, Damien Ernst, and Louis Wehenkel. Extremely randomized trees. Machine Learning, 63(1):3-42, 2006.

[14] Robert M. Haralick, K. Shanmugam, and Its'Hak Dinstein. Textural features for image classification. SMC-3(6):610-621, November 1973.

[15] A. Kadir, L. E. Nugroho, A. Susanto, and P. I. Santosa. Neural network application on foliage plant identification. International Journal of Computer Applications, 29(9):15-22, September 2011.

[16] Abdul Kadir, Lukito Edi Nugroho, Adhi Susanto, and Paulus Insap Santosa. Leaf classification using shape, color, and texture features.
[17] Hanife Kebapci, Berrin Yanikoglu, and Gozde Unal. Plant image retrieval using color, shape and texture features. The Computer Journal, 54(9):1475-1490, 2011.

[18] Yuanqing Lin, Fengjun Lv, Shenghuo Zhu, Ming Yang, Timothée Cour, Kai Yu, Liangliang Cao, and Thomas S. Huang. Large-scale image classification: fast feature extraction and svm training. In CVPR'11: IEEE Conference on Computer Vision and Pattern Recognition.

[19] Timo Ojala, Matti Pietikinen, and David Harwood. A comparative study of texture measures with classification based on featured distributions. Pattern recognition, 29(1):51-59, 1996.

[20] Dan C. Popescu, Radu Dobrescu, and Maximillian Nicolae. Texture classification and defect detection using statistical features. International Journal of Circuits, Systems and Signal Processing, 1(1):79-84, 2007.

[21] Subhra Pramanik, Samir Kumar Bandyopadhyay, Debnath Bhattacharyya, and Tai hoon Kim. Identification of plant using leaf image analysis. Springer Signal Processing and Multimedia Communications in Computer and Information Science, 123:291-303, 2010.

[22] S. K. Prof Shah. Image classification based on textural features using artificial neural network (ANN).

[23] Elio Ramos and Denny S. Fernndez;. Classification of leaf epidermis micrographs using texture features. International Journal of Ecoinformatics and Computational Ecology, Ecological Informatics, 4::177-181, 2009.

[24] E. Sandeep Kumar. Leaf color, area and edge features based approach for identification of indian medicinal plants. Indian Journal of Computer Science and Engineering (IJCSE), 3(3):436-442, Jun-Jul 2012.

[25] Junichi Tsujii Tsuruoaka, Yoshimasa and Sophia Ananiadou. Stochastic gradient descent training for 11-regularized log-linear models with cumulative penalty. In ACL-IJCNLP 2009, pages 477-485, 2009.

[26] Stephen Gang $\mathrm{Wu}$, Forrest Sheng Bao, Eric You Xu, Yu-Xuan Wang, Yi-Fan Chang, and Qiao-Liang Xiang. A leaf recognition algorithm for plant classification using probabilistic neural network. IEEE International Symposium on Signal Processing and Information Technology, pages 11-16, 2007. 
Table 1. Sample Feature Values

\begin{tabular}{|c|c|c|c|c|c|c|}
\hline Leaf & $\mu_{0}$ & $\mu_{2}$ & $\mu_{3}$ & $\sigma$ & $\epsilon$ & $\mu_{L}$ \\
\hline 0 & 50.18 & 663.99 & 0.58 & 28.1 & 5.74 & 118.98 \\
1 & 64.48 & 353.43 & -0.47 & 25.76 & 5.60 & 117.80 \\
2 & 106.71 & 496.96 & -0.24 & 16.30 & 3.31 & 106.68 \\
3 & 115.52 & 789.49 & -0.01 & 19.78 & 2.26 & 113.03 \\
4 & 99.46 & 458.54 & -0.02 & 21.41 & 3.28 & 101.83 \\
\hline
\end{tabular}

Table 2. Preprocessing with category $\mathrm{C1}$

\begin{tabular}{|l|c|c|c|c|c|c|}
\hline Filter & SGD & kNN & SVM & DT & ET & RF \\
\hline HE & 33.3 & 38.7 & 37.3 & 29.3 & 32.0 & 41.3 \\
AHE & 33.3 & 37.3 & 24.0 & 36.0 & 33.0 & 41.3 \\
CLAHE & 33.3 & 34.7 & 24.0 & 33.3 & 34.7 & 38.7 \\
LBP & 48.0 & $\mathbf{4 9 . 3}$ & 42.7 & 33.3 & 34.7 & 41.3 \\
\hline
\end{tabular}

Table 3. Preprocessing with category $\mathrm{C2}$

\begin{tabular}{|l|c|c|c|c|c|c|}
\hline Filter & SGD & kNN & SVM & DT & ET & RF \\
\hline HE & 32.0 & 40.0 & 37.3 & 29.3 & 26.7 & 32.0 \\
AHE & 34.7 & 38.7 & 24.0 & 36.0 & 30.7 & 37.3 \\
CLAHE & 32.0 & 34.7 & 25.3 & 33.3 & 37.3 & 34.7 \\
LBP & $\mathbf{4 8 . 0}$ & $\mathbf{4 8 . 0}$ & 44.0 & 33.0 & 36.0 & 41.3 \\
\hline
\end{tabular}

Table 4. Preprocessing with category $\mathrm{C3}$

\begin{tabular}{|l|c|c|c|c|c|c|}
\hline Filter & SGD & kNN & SVM & DT & ET & RF \\
\hline HE & 33.3 & 28.0 & 26.7 & 29.3 & 28.0 & 32.0 \\
AHE & 45.3 & 44.0 & 40.0 & 41.3 & 36.0 & 41.3 \\
CLAHE & 44.0 & 45.3 & 41.3 & 41.3 & 32.0 & 42.7 \\
LBP & 44.0 & 42.7 & 42.7 & 33.3 & $\mathbf{5 0 . 7}$ & 37.3 \\
\hline
\end{tabular}

Table 5. Preprocessing with category $\mathrm{C4}$

\begin{tabular}{|l|c|c|c|c|c|c|}
\hline Filter & SGD & kNN & SVM & DT & ET & RF \\
\hline HE & 38.7 & 29.3 & 36.0 & 36.0 & 30.7 & 30.7 \\
AHE & 53.3 & $\mathbf{5 6 . 0}$ & 38.7 & 46.7 & 46.7 & 41.3 \\
CLAHE & 49.3 & $\mathbf{5 6 . 0}$ & 40.0 & 49.3 & 38.7 & 52.0 \\
LBP & 44.0 & 42.7 & 42.7 & 33.3 & 38.7 & 37.3 \\
\hline
\end{tabular}

Table 6. Preprocessing with category C5

\begin{tabular}{|l|c|c|c|c|c|c|}
\hline Filter & SGD & kNN & SVM & DT & ET & RF \\
\hline HE & 56.0 & 46.7 & 49.3 & 52.0 & 46.7 & 57.3 \\
AHE & 52.0 & 64.0 & 41.3 & 53.3 & 57.3 & 58.7 \\
CLAHE & 56.0 & $\mathbf{6 6 . 7}$ & 41.3 & 60.0 & 62.7 & 62.7 \\
LBP & 52.0 & 57.3 & 46.7 & 48.0 & 46.7 & 56.0 \\
\hline
\end{tabular}

Table 7. 'No Preprocessing(NP)'

\begin{tabular}{|c|c|c|c|c|c|c|}
\hline Category & SGD & kNN & SVM & DT & ET & RF \\
\hline C1 & 58.7 & 88.0 & 73.3 & 82.7 & 86.3 & 81.3 \\
C2 & 57.3 & 88.0 & 58.7 & 82.7 & 74.7 & 84.0 \\
C3 & 60.0 & 90.7 & 76.0 & $\mathbf{9 4 . 7}$ & 70.7 & 92.0 \\
C4 & 89.3 & $\mathbf{9 4 . 7}$ & 89.3 & 92.0 & 78.7 & 93.3 \\
C5 & $\mathbf{9 4 . 7}$ & 93.3 & 92.0 & 93.3 & 88.0 & 93.3 \\
\hline
\end{tabular}

Table 8. Recognition and Error of C3 using DT

\begin{tabular}{|c|c|c|c|c|}
\hline Leaf & Support & Prediction & $\begin{array}{c}\text { Recognition } \\
\text { Rate }(\%)\end{array}$ & Error \\
\hline 0 & 17 & 16 & 94 & 0.06 \\
1 & 14 & 14 & 100 & 0 \\
2 & 12 & 9 & 75 & 0.25 \\
3 & 17 & 17 & 100 & 0 \\
4 & 15 & 15 & 100 & 0 \\
\hline
\end{tabular}

Table 9. Recognition and Error of $\mathrm{C} 4 \mathrm{using} \mathrm{kNN}$

\begin{tabular}{|c|c|c|c|c|}
\hline Leaf & Support & Prediction & $\begin{array}{c}\text { Recognition } \\
\text { Rate(\%) }\end{array}$ & Error \\
\hline 0 & 17 & 16 & 94 & 0.06 \\
1 & 14 & 14 & 100 & 0 \\
2 & 12 & 10 & 83 & 0.17 \\
3 & 17 & 17 & 100 & 0 \\
4 & 15 & 14 & 93 & 0.07 \\
\hline
\end{tabular}

Table 10. Recognition and Error of C5 using SGD

\begin{tabular}{|c|c|c|c|c|}
\hline Leaf & Support & Prediction & $\begin{array}{c}\text { Recognition } \\
\text { Rate }(\%)\end{array}$ & Error \\
\hline 0 & 17 & 17 & 100 & 0 \\
1 & 14 & 13 & 93 & 0.07 \\
2 & 12 & 11 & 92 & 0.08 \\
3 & 17 & 16 & 94 & 0.06 \\
4 & 15 & 14 & 93 & 0.07 \\
\hline
\end{tabular}

Table 11. Comparison with other methods

\begin{tabular}{|l|c|c|}
\hline Methods & $\begin{array}{c}\text { Recognition } \\
\text { Rate(\%) }\end{array}$ & Error Rate \\
\hline Max-Scale in [17] & 61.8 & 0.38200 \\
Patch-Based in [17] & 50 & 0.50000 \\
Random in [17] & 74 & 0.26000 \\
SVM in [3] & 88.23 & 0.11770 \\
ANN in [3] & 84.59 & 0.15410 \\
PNN in [16] & 93.437 & 0.06563 \\
Proposed method & 94.7 & 0.05300 \\
\hline
\end{tabular}

